\title{
Review of: "Botanical formulation, TADIOS, alleviates lipopolysaccharide (LPS)-Induced acute lung injury in mice via modulation of the Nrf2-HO-1 signaling pathway"
}

\author{
Mingli Peng ${ }^{1}$ \\ 1 Chongqing Medical University
}

Potential competing interests: The author(s) declared that no potential competing interests exist.

This article verified the potential ability of the botanical formulation TADIOS for the treatment of acute respiratory distress syndrome and explored its mechanism of action, which has certain reference value. In recent years, traditional herbal preparations have shown a certain advantage in the treatment of clinical diseases. However, there is no safe and effective drugs currently in the clinical practice of ARDS. The discovery of TADIOS will promote the development of safe and effective drugs for the treatment of ARDS. This article verified that TADIOS inhibited the LPS-mediated expression of pro-inflammatory cytokines and oxidative stress, and activated the Nrf2-HO-1 axis to alleviate acute lung injury in mice. However, since herbal preparations have a multi-target characteristic, it is recommended that the authors could combine transcriptional analysis to more fully understand the molecular targets of TADIOS in subsequent research. 